\title{
Modélisation du charriage en écoulement fluvial
}

\author{
J. Allard P. Chee \\ EDF - Laboratoire national d'hydraulique
}

\section{But et cadre de l'étude}

Cette action s'inscrit dans le cadre de la Convention du 23 juillet 1965 entre le Laboratoire national d'hydraulique d'EDF d'une part et les Ministères (Environnement, Service technique central des Ports maritimes et des Voies navigables) d'autre part.

Le but du programme d'étude est de construire un modèle mathématique monodimensionnel permettant de prévoir le devenir d'un lit fluvial soumis à l'érosion par charriage.

Dans un premier temps, la tentative de calage sur l'évolution du lit du Rhin, mesurée en nature, s'est révélée infructueuse du fait de la complexité des phénomènes [1] et [2].

Un canal d'essais de $50 \mathrm{~m}$ de long a été construit pour rechercher, dans un cas schématique, les paramètres prépondérants du problème. Les premiers essais ont été réalisés en granulométrie uniforme, avec deux types de matériau : bakélite puis sable. Ils ont permis de résoudre un grand nombre des problèmes liés à la mise en œuvre du dispositif expérimental. Cette série d'essais essentiellement axée sur la loi de chargement a relativisé l'importance de ce phénomène et a montré l'importance capitale de bien appréhender la rugosité.

C'est pourquoi, lors de la dernière série d'essais, l'accent a été porté sur la détermination des coefficients de rugosité. D'autre part ces essais réalisés avec trois classes de sable et des mélanges ont permis d'apprécier l'influence de l'étendue granulométrique sur le transport solide.

\section{Le canal expérimental}

La longueur des essais à réaliser, la précision et la patience nécessaires pour obtenir des résultats tangibles ont conduit à automatiser le plus possible le modèle. Le canal expérimental est donc une installation dont la mise en place et le pilotage ont demandé d'importants efforts. Il a une longueur utile de $50 \mathrm{~m}$, une largeur de $0,4 \mathrm{~m}$ et une profondeur de $0,5 \mathrm{~m}$.

Il permet de représenter des lois de débit liquide, de hauteur d'eau aval, de débit solide amont, et de réaliser des mesures de la cote des fonds et des hauteurs d'eau sans interruption des essais (sonde à ultrasons), du débit solide par prélèvements aval, et du volume érodé ou déposé par cubatures entre relevés par la sonde à ultrasons.

Un calculateur permet de piloter l'installation, y compris le déclenchement des mesures [3].

\section{Résistance hydraulique. Coefficient de rugosité}

\subsection{Généralités}

Dans le cas de notre canal à fonds mobiles, où plus généralement de canaux avec berges fixes, la rugosité globale du lit $k$ est fonction de la rugosité du revêtement des berges $k_{p}$ et de celle du fond du lit $k_{s}$; elle en est déduite par la méthode d'Einstein de composition des rugosités [4] et [5].

\section{Modeling of bed load sediment transport for fluvial flow}

The mathematical modeling choices of bed load transport in rivers must be confirmed by precise data. Collection of field data is long and difficult. Besides, the parameters of natural flows, such as discharge cannot be controlled. For these reasons a $50 \mathrm{~m}$ long flume was set up. It makes possible with the help of precise data, no study independantly some of the important physical phenomena involved in bed load transport, such as hydraulic resistance, influence of non uniformity of grain-size, spatial lag effects. This study has been carried out as part of the convention EDF-Ministeres of July 23, 1965. 
D'autre part la rugosité du fond d'un lit constitué de granulats mobiles est définie, d'une part par la taille des grains de sédiments qui la tapissent («rugosité de peau »), d'autre part par les ondulations du fond (ride, dunes...), de tailles en général bien supérieures à celle des grains ("rugosité de formes") [6].

Le coefficient de frottement sur le fond peut donc formellement se décomposer en $k_{s}=f\left(k_{s}^{\prime}, k_{s}^{\prime \prime}\right) ; k_{s}^{\prime}$ étant le frottement dû aux grains, $k_{s}^{\prime \prime}$ le frottement dû aux formes du lit (lié à un coefficient de traînée de l'écoulement sur ces ondulations). Sur des lits de graviers ou de galets, on observe rarement des dunes (quelquefois des "barres" rocheuses). Les pertes de l'écoulement par frottement sont essentiellement dues aux aspérités du lit, à la rugosité de peau : $k_{s} \approx k_{s}^{\prime}$. Par contre, rides et dunes sont des profils caractéristiques des lits sableux. Elles induisent en général une résistance à l'écoulement, un frottement, bien supérieur à celui induit par les seuls grains : $k_{s} \approx k_{s}^{\prime \prime}$. L'intensité du transport solide est fonction de la puissance dissipée par l'écoulement pour mettre en mouvement les grains de sédiment. Cette puissance est une fraction de la puissance dissipée par l'écoulement par frottement sur le fond.

Plus l'écoulement dissipera de puissance à vaincre la résistance hydraulique des ondulations du lit, moins il gardera de "puissance efficace " pour transporter des sédiments. Il est donc essentiel, pour un calcul de transport solide, de pouvoir prévoir le frottement global $k_{s}=f\left(k_{s}^{\prime}, k_{s}^{\prime \prime}\right)$.

Diverses formules empiriques permettent de calculer directement la rugosité du fond à l'aide des données des paramètres moyens de l'écoulement, ou des caractéristiques du sédiment composant le fond, ou des deux.

On relie en général la rugosité de peau au diamètre représentatif des fractions granulométriques les plus grossières du lit alluvial.

(ex. : formule de Manning-Strickler $-k_{s}^{\prime}=\frac{26}{\left(d_{90}\right)^{1 / 6}}$

ou de Ramette $\quad k_{s}^{\prime}=\frac{26,4}{\left(d_{50}\right)^{1 / 6}}\left(\frac{d_{50}}{h}\right)^{1 / 24}$

avec $h=$ profondeur d'eau).

Les formules ont toutes été calibrées sur des lits plats, souvent sur des lits où les aspérités étaient fixes.

La rugosité de formes est, elle, en général estimée après prévision (toujours par le moyen de relations semiempiriques) de la hauteur et de la raideur des ondulations du lit.

\subsection{Etude sur le canal}

L'ensemble de ses constatations nous ont conduit à adopter la démarche suivante :

- canal sans sédiment, berges et fonds fixes de même rugosité :

- détermination de la rugosité de paroi $k_{p}$,

- introduction du sédiment :

- à partir de lignes d'eau sans charriage, détermination de la rugosité de peau $k_{s}^{\prime}$,
- suivi au cours de l'essai de l'évolution du coefficient de rugosité du lit parallèlement à celles des formes se développant sur le fond.

\subsection{Résultats}

La figure 1 représente l'évolution de $k_{s}$ au cours d'un essai. En présence de dunes, les fonds sont modélisés pour cet étalonnage, par un profil moyen passant à mihauteur de ces dunes. On peut remarquer que :

- la rugosité de peau en début d'essai est plus grande que celle en fin d'essai (i.e. le coefficient de Strickler est plus faible). Les fonds sont plats pour les deux cas mais l'arrangement des granulats est différent en fin d'essai après un tassement naturel par l'écoulement correspondant à une meilleure imbrication des grains entre eux que celle créée artificiellement lors de la réfection des fonds et qui présente des aspérités plus marquées. Cette constatation se confirme sur tous les essais. Notons que dans l'hypothèse d'une extrapolation à des données nature, la rugosité de peau à prendre en compte se rapproche bien évidemment de celle de fin d'essai correspondant à une fin de cycle de charriage (passage d'une crue par exemple) ;

- la rugosité augmente lors de l'apparition des formes du lit ( 0 à 24 h sur la figure 1$)$ puis diminue lors de leur disparition et au retour à un lit plat.

\section{Influence de la granulométrie sur le transport solide}

\subsection{Présentation des essais}

Tous les essais ont été effectués en régime hydraulique permanent ou plus précisément avec un débit et une cote aval maintenus constants pendant la totalité de l'essai et en l'absence d'alimentation en matériau solide à l'amont.

Le profil en long initial est à contre-pente ce qui permet d'obtenir (fig. 2) :

- des contraintes de cisaillement inférieures à la contrainte critique sur les premiers mètres des fonds mobiles (fort tirant d'eau, pas de charriage) ;

- début de charriage en un point de la contre-pente ; - charriage sur l'aval.

Les essais durent entre 24 h et 200 h. Ils sont arrêtés en principe après la disparition des dunes et l'apparition des fonds plats sur la partie aval du canal. Cependant pour les charriages importants, une fosse apparaît immédiatement après le seuil amont. Dans ce cas, les essais sont arrêtés avant que la fosse devienne trop importante pour que son effet sur le charriage soit négligé. La stabilisation du fond après l'arrêt complet du charriage n'a jamais été atteinte, ce processus étant très lent.

Trois classes de sable ont été utilisées ayant des diamètres $d_{50}$ de $1,1,5,2 \mathrm{~mm}$ : classes $\mathrm{A}, \mathrm{B}, \mathrm{C}$ respectivement. Les essais ont été réalisés sous les mêmes conditions hydrauliques (débit et cote aval) avec successivement les classes $B, A$ et $C$ puis avec les mélanges $A+C$ et $A+B+C$ en égales proportions. Pour ces deux mélanges, le $d_{50}$ est proche de $1,5 \mathrm{~mm}$ comme pour 


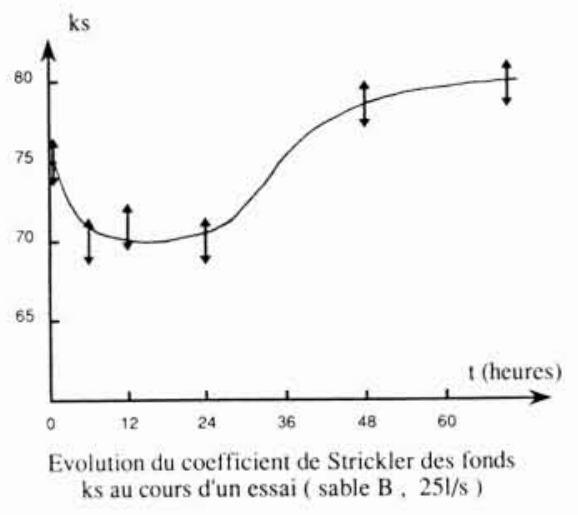

1.

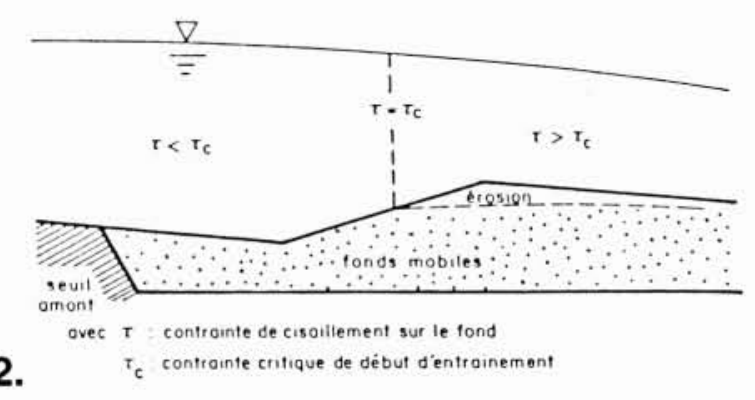

le sable $B$ (fig. 3). Seule change l'étendue granulométrique.

\subsection{Evolution des fonds}

Quantitativement, le profil en long moyen des fonds sableux après $24 \mathrm{~h}$ d'essais est plus bas pour le sable $A$ que pour le $B$, lui-même plus bas que le $C$. Ceci est évidemment dû à la taille plus fine des sédiments qui facilite le charriage.

Les différences sont aussi qualitatives car les modes de charriage ne sont pas identiques pour les trois sables. Alors que le charriage se fait par bancs successifs pour les sédiments les plus fins, il s'effectue avec un lit plat pour les grains les plus gros. Le charriage du sable $B$ s'effectue par bancs à l'aval et par lit plat à l'amont.

La figure 4 ne permet pas d'affirmer l'existence de différences quantitatives pour le charriage après $24 \mathrm{~h}$ d'essais, entre les sables $A+B+C, B$ et $A+C$. Cependant des différences qualitatives apparaissent. En effet le charriage du sable $A+B+C$ s'effectue par des dunes longues et complexes avec des petites dunes sur les grosses, alors que les dunes formées par les sables de granulométries uniformes étaient très régulières. Les dunes observées pour le charriage du sable $A+C$ sont encore différentes, elles sont longues et complexes comme celles du sable $A+B+C$ mais avec une pente beaucoup plus abrupte. En résumé nous pouvons faire les observations suivantes :

Sable A : Apparition de dunes régulières et importantes sur tout le canal. Elles se propagent tout au long de l'essai sans s'amortir. On arrête l'essai lorsque l'effet dû à la fosse amont devient significatif. Le charriage est très important.

Sable B: Apparition de dunes régulières sur la partie aval du canal seulement. Ces dunes disparaissent ensuite en cours d'essai lorsque l'érosion progressive accroît la hauteur d'eau.
COURBE GRANULOMETRIQUE DES

DIFFERENTES CLASSES DE SABLE

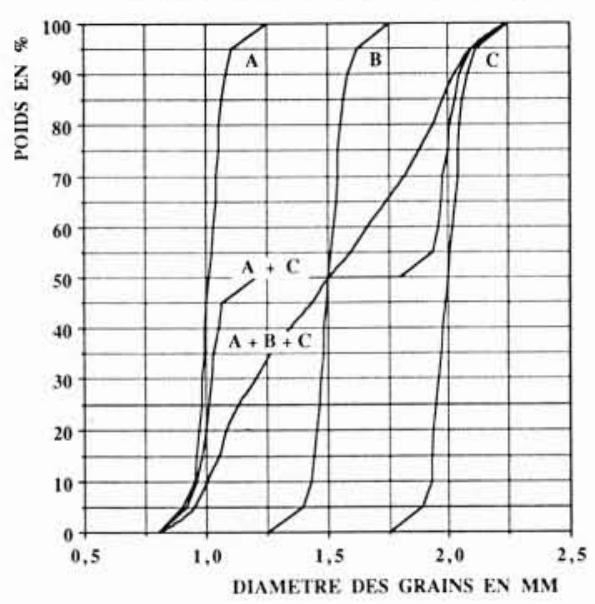

3.

ESSAIS EN REGIME PERMANENT , SANS RECIRCULATION

$Q=20 \mathrm{t} / \mathrm{s}$, Zaval $=117 \mathrm{~mm}$, relevé des fonds après 24 heures.
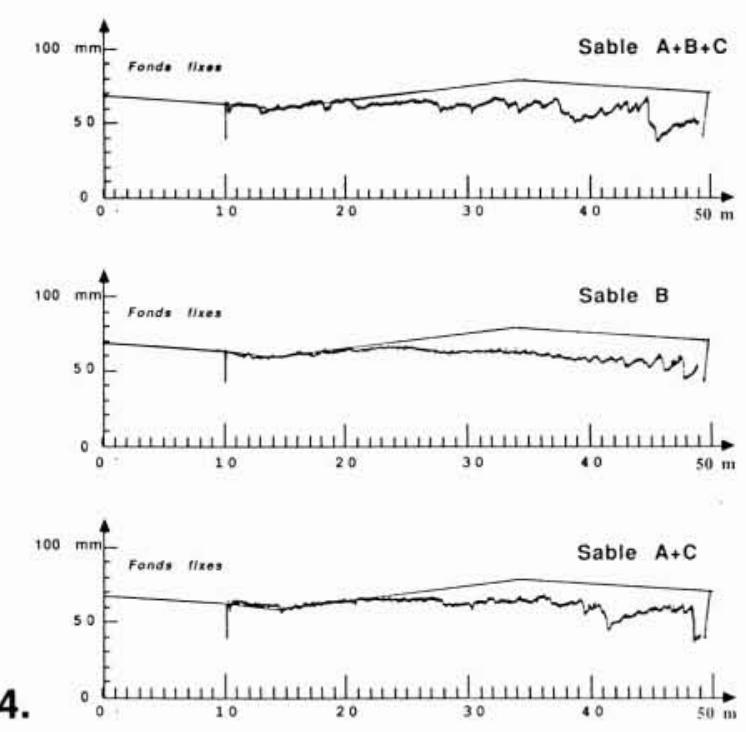

POIDS DE SABLE PRELEVE A L'AVAL, 25L/s

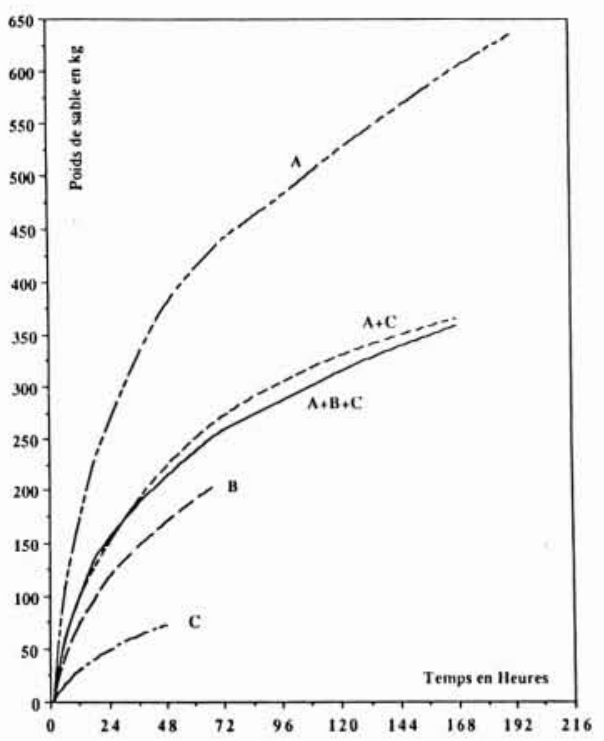

5. 
Sable $A+B+C$ : Apparition de dunes plus grosses et plus irrégulières que pour le sable $B$. Le niveau du fond se situe sensiblement au même niveau que pour le sable $B$. Ici aussi les dunes disparaissent, plus tardivement toutefois.

Sable $A+C$ : Phénomènes très semblables à ceux du sable $A+B+C$. La différence entre les profils d'équilibre pour le sable $B, A+B+C, A+C$ est très faible, elle n'est pas détectable visuellement.

Sable $C$ : Les contraintes près du fond sont insuffisantes pour provoquer l'apparition de dunes, l'érosion s'effectue régulièrement sur la partie en pente descendante de l'aval. L'érosion est nettement plus faible que pour les autres catégories de sables.

\subsection{Importance du charriage}

Si l'on ne note pas de différence nette pour les poids charriés entre les deux mélanges $A+B+C$ et $A+C$, par contre, et c'est là le point essentiel, on constate (fig. 5) une différence entre les mélanges et le sable homogène $B$.

L'ordre de grandeur de cette différence est de 15 à $25 \%$. Elle apparaît quel que soit le procédé d'évaluation utilisé (prélèvements aval, bilans établis avec les relevés de la sonde à ultra-sons) et ne peut donc pas être expliquée par l'imprécision des mesures.

On a vu (fig. 4) que pour les mélanges $A+B+$ $C$ et $A+C$, les dunes sont plus importantes, plus irrégulières et persistent davantage que pour le sable $B$. Ces dunes augmentent la rugosité globale. Il semble qu'en effet un fond plus rugueux augmente la pente de la ligne d'énergie, d'où une possibilité de transport plus grande et un charriage plus fort. Ceci peut expliquer la différence de sable transporté.

Affiner cette hypothèse impliquerait donc de comparer le charriage pour deux granulométries (par exemple $B$ et $A+C$ ) dans le cas de lit plat et dans le cas de dunes. Cependant dans le $1^{\text {er }}$ cas, le charriage étant faible les différences seront difficiles à mettre en évidence.

Il semble néanmoins souhaitable d'effectuer de nouveaux essais sur ce point important avec la présence de fonds plats et de dunes.

\subsection{Tri granulométrique}

Des analyses granulométriques ont été réalisées au moyen de tamis successifs aux mailles de plus en plus fines. Ces tamis sont placés sur un vibreur afin d'obtenir un tamisage semblable pour toutes les analyses.

Tout d'abord plusieurs prélèvements ont été analysés avant essai pour chaque catégorie de sable. Ceci permet d'avoir la composition réelle des sables qui diffère légèrement des courbes théoriques de la figure 3.

De plus, pour les essais effectués avec les mélanges, nous avons réalisé des analyses en cours d'essai sur le sable recueilli à l'aval. Nous avons également analysé en fin d'essai différents points de la surface du canal.

Les résultats de ces prélèvements montrent une composition sensiblement identique pour les prélèvements aval comme pour ceux de surface, à l'état initial, aux erreurs de mesures près.

Aucun tri significatif n'a donc été mis en évidence lors du charriage au moyen de ces essais.

\section{Conclusion}

Les points essentiels dégagés lors de cette étude sont les suivants :

- on a pu évaluer l'évolution des coefficients de rugosité des fonds au cours d'un essai. La rugosité globale dépend dans le canal fortement de la présence de dunes;

- on observe une influence notable de l'étendue granulométrique sur le transport solide : les volumes transportés (et donc le débit solide) sont plus importants pour un mélange que pour un matériau homogène de même diamètre moyen ;

- le phénomène de tri granulométrique n'a pas été mis en évidence. Il serait intéressant d'effectuer des essais avec une granulométrie plus étendue pour se rendre compte de la façon dont apparaît un tri granulométrique et éventuellement le pavage.

En continuité avec ces travaux, un programme d'études a été défini avec des moyens plus importants et un objectif plus ambitieux. Le projet, regroupant les compétences du LNH et du LHF/CEFRHYG, prend ainsi un caractère national.

\section{Bibliographie}

[1] UAN M. - Etude d'une modélisation du transport solide en rivière. Rapport $\mathrm{n}^{\circ}$ 1, EDF E43/83.36.

[2] UAN M. - Etude d'une modélisation du transport solide en rivière. Rapport $\mathrm{n}^{\circ}$ 2, EDF E43/83.47.

[3] Allard J., Chauvel L. - Modélisation du transport solide en rivière. EDF E43/85.10.

[4] AllaRd J. - Modélisation du transport solide en rivière. Canal 24: Essais en régime permanent. EDF E43/85.47.
[5] Allard J. - Modélisation du transport solide en rivière. Canal 24: Essais en régime permanent et en granulométrie uniforme (suite). EDF E43/86.49.

[6] SıмON L. - Transport solide de sédiments de granulométrie non uniforme. EDF E43/86.28.

[7] Allard J., Chee P. - Modélisation du transport solide en rivière. Canal 24: Essais en régime permanent pour des granulométries uniformes et non uniformes. EDF E43/88.12. 